\title{
Comparative assessment of different approaches for the use of CAD geometry in Monte Carlo transport calculations
}

\author{
B. Weinhorst ${ }^{1}$, U. Fischer ${ }^{1}$, L. Lu ${ }^{1}$, Y. Qiu ${ }^{1}$, P. Wilson ${ }^{2}$
}

${ }^{1}$ Karlsruhe Institute of Technology. Institute for Neutron Physics and Reactor Technology $\quad 2$ University of Wisconsin-Madison, Computational Nuclear Engineering Research Group

\section{Objectives}

- Single (unique) CAD geometry: ITER CAD benchmark model.

- Three different approaches for use of CAD geometry in MC transport calculations:

McCad: Current standard approach, conversion of CAD geometry into MCNP representation using McCad developed at KIT.

MCNP6\&TT: Using MCNP6's unstructured mesh geometry feature, meshing of CAD geometry with the tesselationtetrahedralization (TT) approach developed at KIT.

DAGMC: Direct particle tracking on the CAD geometry using a patched version of MCNP developed at UW-Madison.

- Comparison with respect to performance and user-friendliness:

Installation (Installation guide, needed software, installation complexity).

Model preparation (repairing geometry error, time needed, user expertise).

Computation performance (calculation speed, accuracy)

$$
\text { McCad }
$$

MCNP6\&TT

- Good installation guide

Only one additional software package required.

- Open Source software (except MCNP).

- Simple installation.

Model

preparation

performance

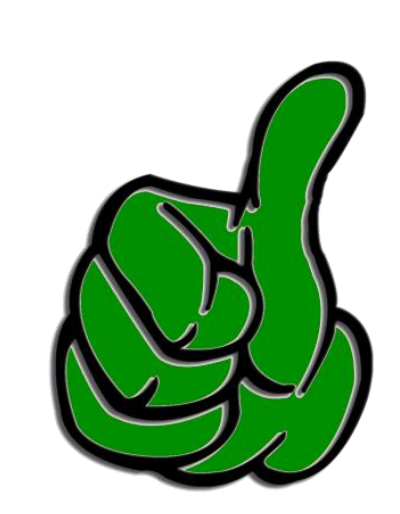

Fastest calculation.

- Current standard approach

for MCNP calculations,

chosen as reference. resolution

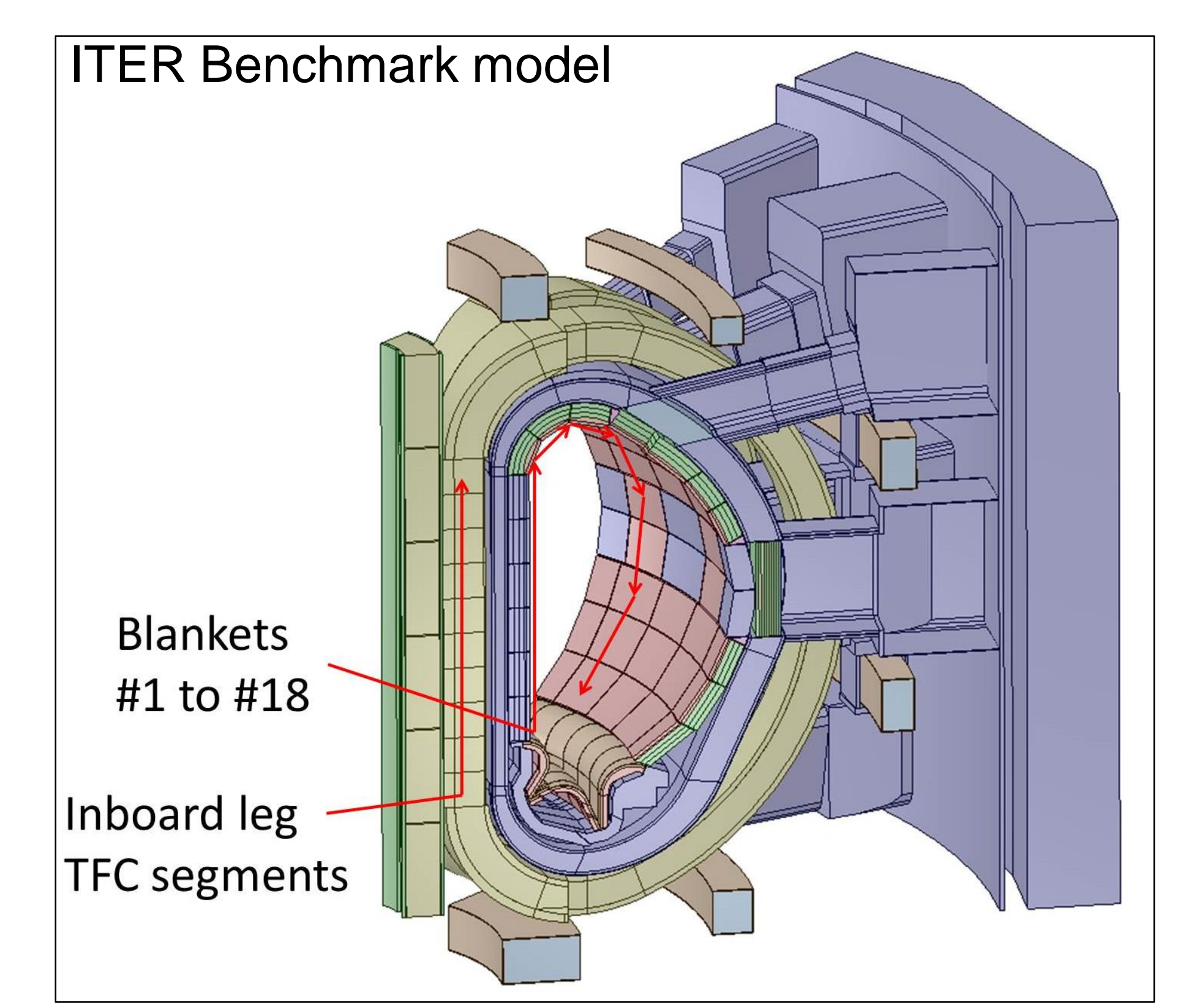

DAGMC
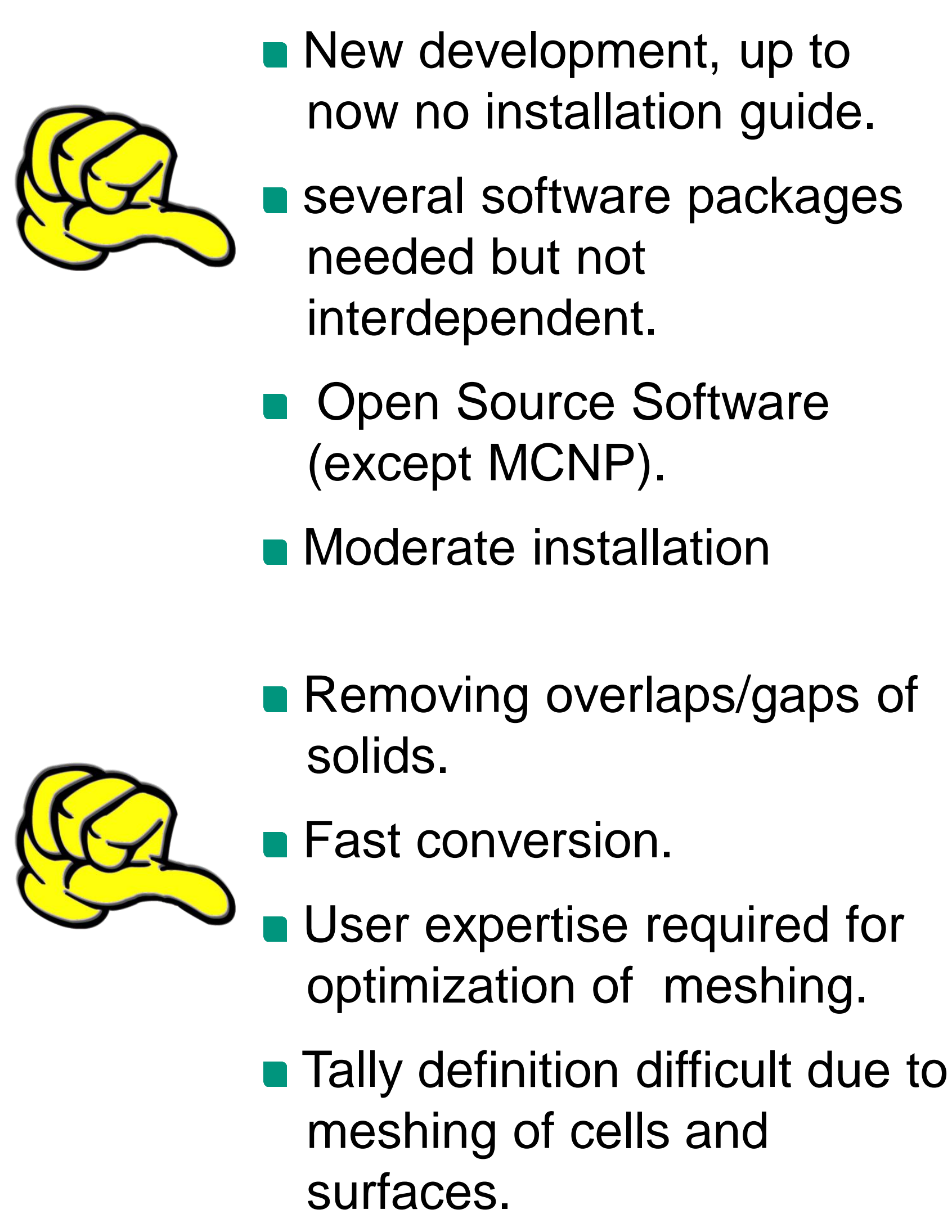

Slowest calculation; speed depends mainly on mesh

- Superimposed mesh gives large deviation for deep penetration calculations

- Cell tallys agree with McCad results within statistical errors.
- Installation guide available,

but not sufficient.

- Interdependent software packages. Dependent on specific, older versions. Cubit and MCNP under license control.

- Complex installation.

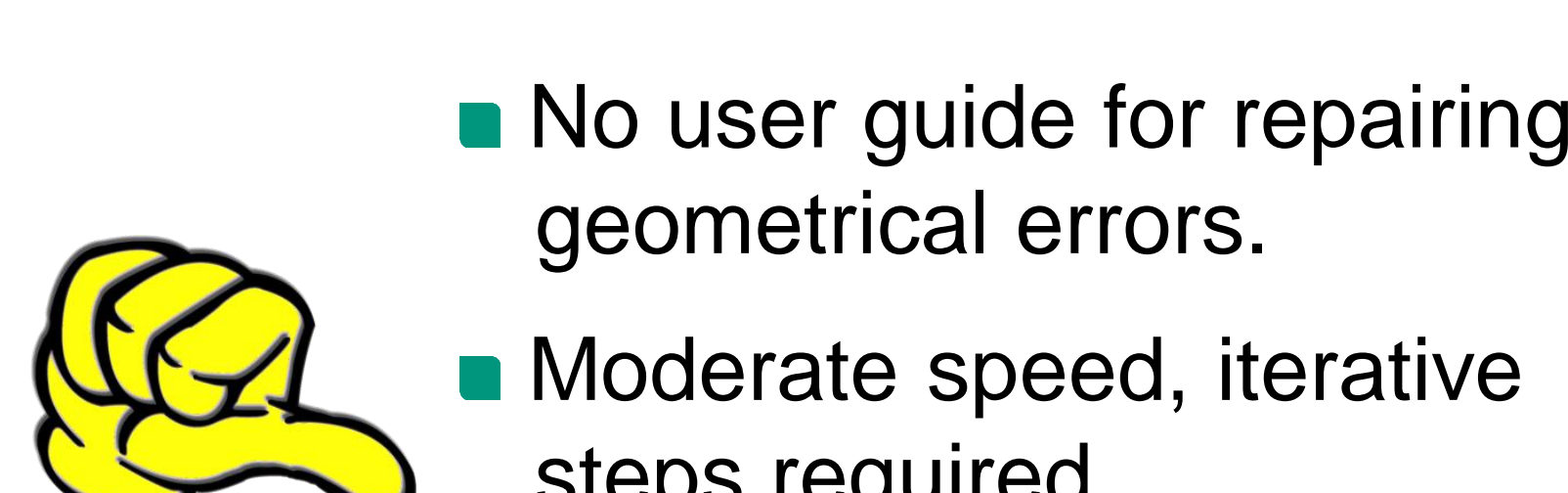

Moderate speed, iterative

quired.

- User expertise essential.

- Tally definition very convenient, more userfriendly than standard MCNP.

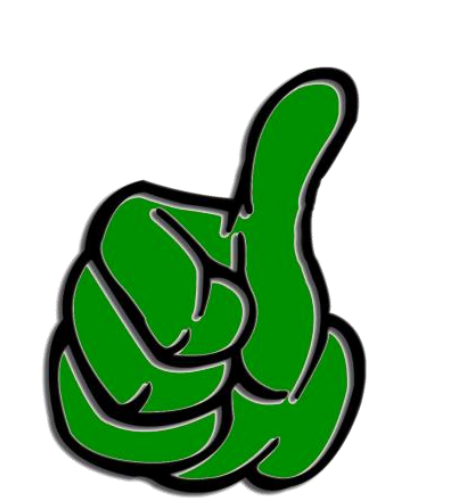

- Moderate calculation speed

- If model preparation done correctly, very good agreement with McCad results.

- First wall cell tallys within 1\% of McCad results.

Comparison of nuclear heating in the inboard leg of the Toroidal Field Coil (TFC)

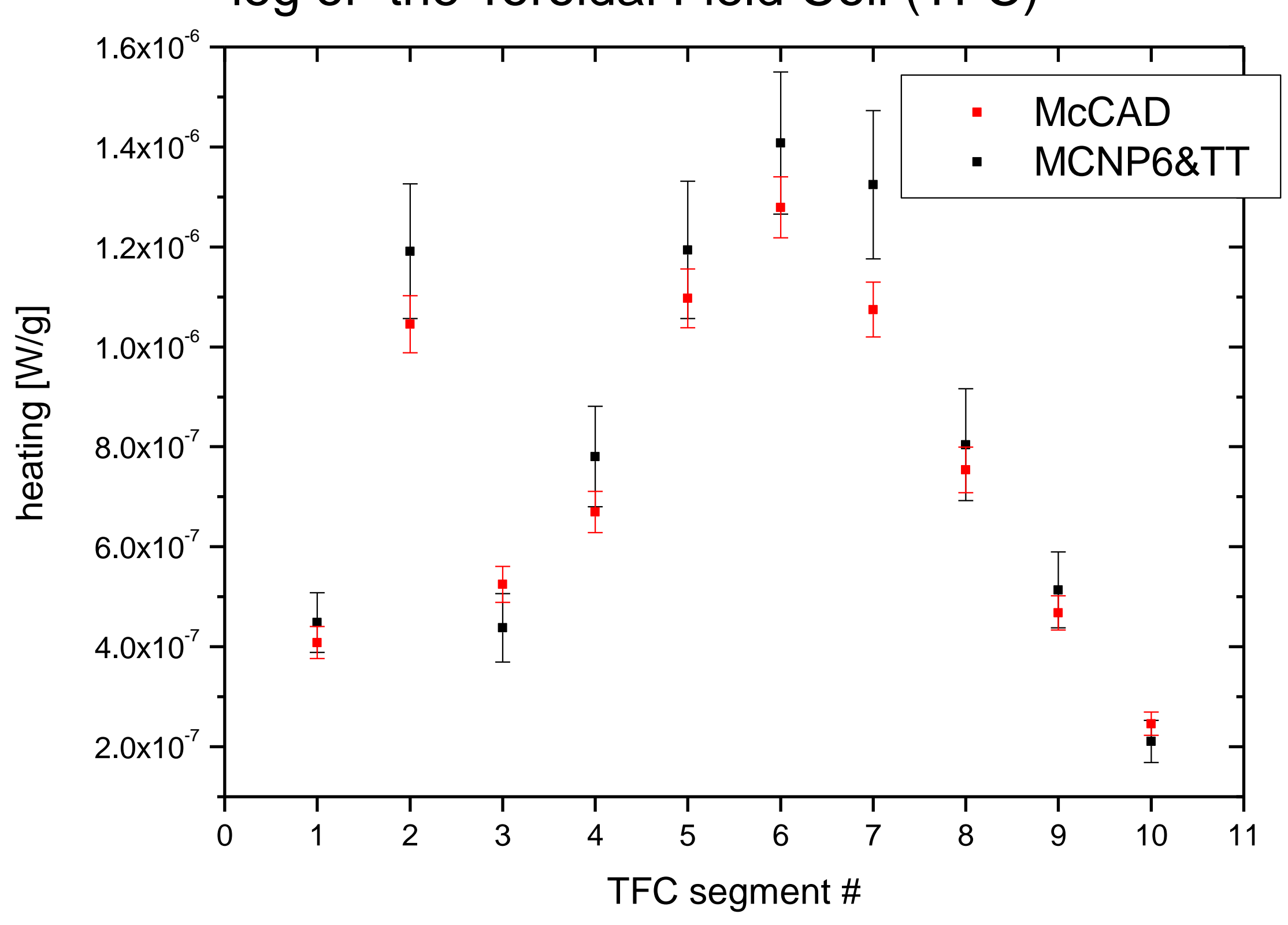

Comparison of $14 \mathrm{MeV}$ neutron flux at First Wall (FW)

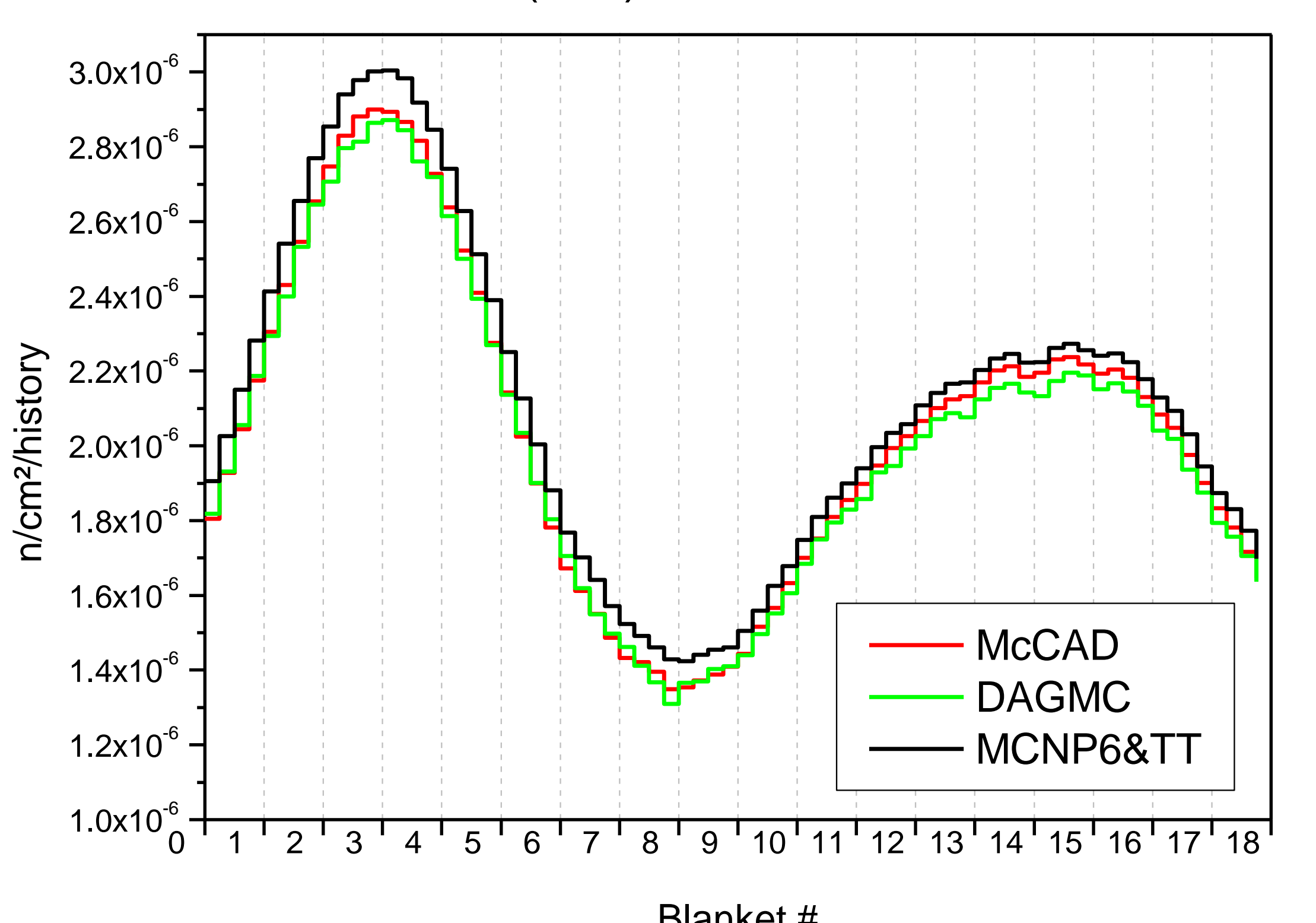

\section{Conclusions}

- McCad most useful for simple models or if model preparation has to be done only once. Small changes to the geometry can be done directly in the MCNP input file. - MCNP6\&TT model preparation extremely fast and reliable. Problematic with regard to nuclear responses; for meshtally the deviation to McCad approach lager than statistical error. Statistical error in general larger than for McCad approach (same number of histories).

- DAGMC most useful for complex models that need to be changed regularly. 